\title{
Surgery was associated with greater long term treatment success than wrist splinting in carpal tunnel syndrome
}

\author{
Gerritsen AA, de Vet HC, Scholten RJ, et al. Splinting vs surgery in the treatment of carpal tunnel syndrome: a randomized \\ controlled trial.JAMA 2002;288:1245-51.
}

\section{QUESTION: In patients with carpal tunnel syndrome (CTS), what is the short term and long term effectiveness of wrist splinting and surgery?}

\section{Design}

Randomised (allocation concealed*), blinded \{data collectors, outcome assessors, and data analysts $\} \uparrow, *$ controlled trial with 18 months of follow up.

\section{Setting}

13 neurological outpatient clinics in the Netherlands.

\section{Patients}

176 patients $\geq 18$ years of age (mean age 49 y, 81\% women) with clinically and electrophysiologically confirmed idiopathic CTS. Exclusion criteria were previous treatment with splinting or surgery, history of wrist trauma or surgery, underlying causes of CTS, conditions emulating CTS, or severe thenar muscle atrophy. Follow up was $95 \%$ at 1 month and $84 \%$ at 18 months.

\section{Intervention}

89 patients were allocated to wrist splinting during the night for $\geq 6$ weeks, and 87 were allocated to open carpal tunnel release surgery.

\section{Main outcome measures}

Patient self report of treatment success (defined as ratings of "completely recovered" or "much improved" on a 6 point ordinal scale ranging from "completely recovered" to "much worse"); number of nights waking because of symptoms; and severity of the main complaint and paresthaesia (each scored on an 11 point numerical scale, with 0 representing "no symptoms" and 10 representing "very severe symptoms").

\section{Main results}

Analysis was by intention to treat. Treatment success rates did not differ between groups at 1 month but were higher in the surgical group at 18 months (table). At 1 month, patients in the splinting group reported greater improvement in the number of nights/week waking because of symptoms than those in the surgical group (mean improvement $2.0 v 0.8 \mathrm{~d}, \mathrm{p}=0.008$ ) and greater improvement in reducing the severity of paresthaesia at night than those in the surgical group (mean score improvement $2.5 v 1.3, \mathrm{p}=0.02$ ); groups did not differ at 18 months. At 18 months, patients in the surgical group reported greater improvement in reducing the severity of the main complaint than those in the splinting group (mean score improvement $6.2 v 5.0, \mathrm{p}=0.02$ ) and greater improvement in reducing the severity of paresthaesia during the day than those in the splinting group (mean score improvement $5.3 v 4.0, \mathrm{p}=0.01$ ); groups did not differ at 1 month.

\section{Conclusion}

In patients with carpal tunnel syndrome, surgery and wrist splinting did not differ greatly in the short term, but surgery was associated with greater treatment success than splinting in the long term.

*See glossary.

$\dagger$ Information provided by author.

\section{COMMENTARY}

Gerritsen $e t$ al are to be commended for their well designed, randomised trial of CTS. Their conclusions largely mirror results of 1 previous smaller, randomised study. ${ }^{1}$ The methodology was excellent, and the best possible next to doing sham operations. It is important to note that analysis of patients' treatment preferences before randomisation did not affect outcome. However, possible changes of hand use activities (eg, employment and hobbies) were not included in an analysis of the long term outcomes.

Despite these results, several issues should be considered before sending patients immediately to surgery. Firstly, patient selection in this study was restricted. Patients with underlying causes of CTS, a history of wrist trauma or previous treatment, and those with severe thenar atrophy were excluded. These groups account for a large proportion of patients with CTS. Although this makes for a well controlled trial, it does not accurately reflect typical clinical practice. Secondly, although the difference between groups for the primary outcome of success is clinically significant at 18 months, the difference between the groups narrows over time, making study of even longer term outcomes imperative. However, this was mainly caused by a large proportion of patients who received splinting $(41 \%)$ going on to receive surgery.

The study advances the argument around treatment of CTS in favour of surgery in selected patients. Given the limitations of patient inclusion, the lack of accounting for altered hand use, and the potential for serious side effects from surgery (albeit extremely low), treatment still needs to be individualised. The current American Academy of Neurology recommendation of an initial trial of noninvasive therapy still seems to be reasonable for a large number of patients. Richard Barbano, MD, PhD University of Rochester, Rochester, New York, USA

1 Verdugo RJ, Salinas RS, Castillo J, et al. Surgical versus nonsurgical treatment for carpal tunnel syndrome. Cochrane Database Syst Rev 2002;(2):CD001552.

Treatment success of surgery $v$ wrist splinting in carpal tunnel syndrome

\begin{tabular}{|c|c|c|c|c|}
\hline Follow up & Surgery & Splinting & RBR (95\% Cl) & NNH \\
\hline 1 month & $29 \%$ & $42 \%$ & $32 \%$ (-3 to 55$)$ & Not significant \\
\hline & & & RBI (Cl) & NNT (Cl) \\
\hline 18 months & $90 \%$ & $75 \%$ & $20 \%$ (3 to 42 ) & 7 (4 to 39$)$ \\
\hline
\end{tabular}

Source of funding: Health Care Insurance Council of the Netherlands.

For correspondence: $\operatorname{Dr}$ A A Gerritsen, Institute for Research in Extramural Medicine, Amsterdam, The Netherlands. aam.gerritsen.emgo@ med.vu.nl 